\title{
(Inverse) magnetic catalysis in non-relativistic Bose-Einstein condensation
}

\section{Bo Feng*}

School of Physics, Huazhong University of Science and Technology, Wuhan, 430074, China Institute of Particle Physics and Key Laboratory of Quark and Lepton Physics (MOE), Central China Normal University, Wuhan 430079, China

E-mail: bfengdhust.edu.cn

\section{De-fu Hou}

Institute of Particle Physics and Key Laboratory of Quark and Lepton Physics (MOE), Central China Normal University, Wuhan 430079, China

E-mail: houdfemail.cenu.edu.cn

\section{Hai-cang Ren}

Physics Department, The Rockefeller University, 1230 York Avenue, New York, New York 10021-6399, USA

Institute of Particle Physics and Key Laboratory of Quark and Lepton Physics (MOE), Central China Normal University, Wuhan 430079, China

E-mail:

We study the Bose-Einstein condensation of neutral bound pairs in a magnetic field.We found that the condensation temperature shows the magnetic catalysis effect in weak coupling and the inverse magnetic catalysis effect in strong coupling. The different responses to the magnetic field can be attributed to the competition between the dimensional reduction by Landau orbitals in pairing dynamics and the anisotropy of the kinetic spectrum of fluctuations.

9th International Workshop on Critical Point and Onset of Deconfinement - CPOD2014,

17-21 November 2014

ZiF (Center of Interdisciplinary Research), University of Bielefeld, Germany

\footnotetext{
* Speaker.
} 


\section{Introduction}

The behavior of a system consists of charged fermions in a magnetic field had attracted considerable interests in recent years. We investigate a system that share the same physics as chiral magnetic catalysis, the Bose-Einstein Condensation(BEC) of composite Bosons-neutral bound pairs made of two oppositely charged fermions in the presence of an external magnetic field.

The terminology "Magnetic Catalysis" refers to the fact that chiral symmetry is always spontaneously broken at finite magnetic field regardless of the coupling strength $[2,3]$. It would thus be natural to expect a higher transition temperature from the chiral broken phase to the chiral symmetric phase due to magnetic catalysis effect. This is indeed the case within mean-field approximations of effective model studies, it was found that the chiral phase transition is significantly delayed by a nonzero magnetic field even including the $\rho$ meson contribution [4-6]. The recent lattice calculations [7], however, provide surprising results that the chiral pseudo-critical temperature significantly decreases for increasing magnetic field. On the other hand, the chiral condensate increase with increasing magnetic field at low temperature consistent with magnetic catalysis while it turns out to a monotonously decreasing at high temperature [8], which is in apparent conflict with magnetic catalysis and termed as inverse magnetic catalysis evoking an extensive studies [9-16].

While mean field approximation gives sensible results in certain circumstances, fluctuations can break it down, especially in strong coupling domain or in lower dimensions. As was shown in [17] in the absence of magnetic field, a long range order cannot survive at a nonzero temperature in the spatial dimensionality two or less because of the fluctuation of its phase. A long wavelength component of the fluctuation variance goes like $1 / p^{2}$ with $\mathbf{p}$ the momentum, which gives rise to infrared divergence of the momentum integration in two and lower dimensions. The anisotropy introduced by a magnetic field $B \hat{\mathbf{z}}$ renders the long wavelength fluctuation $\sim 1 /\left(p_{z}^{2}+\kappa p_{\perp}^{2}\right)$, with $\kappa$ positive constant between zero and one. Such a distortion of the bosonic spectrum towards dimensionality one $(\kappa \rightarrow 0)$, as a consequence of the dimension reduction of the pairing fermions, would enhance the phase fluctuation. A preliminary study of the Ginzburg-Landau theory of the chiral phase transition [18] reveals the same effect and the Ginzburg critical window gets widened in the presence of magnetic field, indicating the enhancement of the long wavelength fluctuations.

The BEC of bound pairs made of oppositely charged fermions in a magnetic field provides another platform to explore the competition between the enhanced Cooper pairing by Landau orbitals and the enhanced phase fluctuation by the distortion of the bosonic spectrum. Our system corresponds to the BEC limit of the BCS/BEC crossover, which has been studied extensively in the absence of magnetic field for nonrelativistic fermions [19,20] and relativistic ones [21-24]. We found that the critical temperature for the BEC was dramatically affected by the magnetic field exhibiting magnetic catalysis or inverse magnetic catalysis depending on the coupling strength. In the weak coupling domain, where no bound pairs(composite bosons) exist at zero magnetic field, the magnetic catalysis induces bound pairs and thereby a BEC. The critical temperature increases with increasing magnetic field. In the strong coupling domain, where bound pairs exist without magnetic field, an inverse magnetic catalysis was found. The critical temperature decreases as increasing magnetic field, signaling the enhanced fluctuation in a magnetic field.

The rest of the paper is organized as follows: in Section II we lay out the general framework within which we take into account the Gaussian fluctuations. The magnetic field dependence of 
the BEC temperature is investigated in Section IV. Section V is devoted to the conclusions and outlooks. Throughout the paper, we will work in Euclidean space with the four vector represented by $x^{\mu}=(i \tau, \mathbf{x}), q^{\mu}=\left(i \omega_{n}, \mathbf{q}\right)$ with $\omega_{n}$ the Matsubara frequency for bosons $\omega_{n}=2 i \pi n T$ and for fermions $\omega_{n}=(2 n+1) i \pi T$.

\section{General framework}

We consider a system consisting of nonrelativistic fermions of mass $m$ and chemical potential $\mu$ with opposite charge interacting through a short ranged instantaneous attractive interaction. The Hamiltonian density reads

$$
\mathscr{H}\left[\psi, \psi^{\dagger}\right]=\sum_{\sigma= \pm} \psi_{\sigma}^{\dagger}(x)\left[\frac{(-i \nabla+\sigma e \mathbf{A})^{2}}{2 m}-\mu\right] \psi_{\sigma}(x)-g \psi_{+}^{\dagger}(x) \psi_{-}^{\dagger}(x) \psi_{-}(x) \psi_{+}(x) .
$$

where $e>0$ is the charge magnitude carried by each fermions, $g>0$ and $\mathbf{A}$ is the vector potential underlying an external magnetic field, $\mathbf{B}=\nabla \times \mathbf{A}$. To avoid the Meissner effect, only fermions with opposite charges can pair. For the sake of simplicity, we ignore the spin degrees of freedom.

Introducing the standard Hubbard-Stratonovich field $\Delta(x)$ coupled to $\psi_{+}^{\dagger} \psi_{-}^{\dagger}$ and integrating out the fermionic fields, we obtain the parition function

$$
\mathscr{Z}=\mathscr{N} \int \mathscr{D} \Delta^{*}(x) \mathscr{D} \Delta(x) \exp (\mathscr{S}[\Delta(x)]),
$$

with the action $\mathscr{S}$ given by

$$
\mathscr{S}[\Delta]=-\int d \tau d^{3} \mathbf{x} \frac{|\Delta(x)|^{2}}{g}+\operatorname{Tr} \ln G^{-1}\left(x, x^{\prime}\right),
$$

where the inverse propagator in Nambu-Gorkov space is

$$
G^{-1}=\left[\begin{array}{cc}
-\frac{\partial}{\partial \tau}-\frac{(-i \nabla+e \mathbf{A})^{2}}{2 m}+\mu & \Delta(x) \\
\Delta^{*}(x) & -\frac{\partial}{\partial \tau}+\frac{(-i \nabla+e \mathbf{A})^{2}}{2 m}-\mu
\end{array}\right] \delta\left(x-x^{\prime}\right) .
$$

The mean field approximation corresponds to set $\Delta(x)=\Delta_{0}$, which is determined by the saddle point condition $\delta \mathscr{S} / \delta \Delta_{0}=0$. A nontrivial saddle point, $\Delta_{0} \neq 0$, corresponds to a long range order and the superfluidity phase of the system. $\Delta_{0}$ drops to zero at the transition to the normal phase. One then obtains the familiar gap equation

$$
\frac{1}{g}=\frac{1}{2 V} \sum_{k_{y}, k_{z} ; l} \frac{1}{\varepsilon_{k_{z}}+l \omega_{B}-\chi} \tanh \frac{\varepsilon_{k_{z}}+l \omega_{B}-\chi}{2 T_{c}} .
$$

where $l=0,1,2, \ldots$ are the Landau levels and $\varepsilon_{k_{z}}=k_{z}^{2} / 2 m$. We have also defined $\chi=\mu-\omega_{B} / 2$ with $\omega_{B}=e B / m$ the cyclotron frequency. In BCS limit, this equation would be solved to yield the critical temperature with the chemical potential given by that of an ideal Fermi gas at a given density. In BEC limit, however, the role is reversed [20]. Eq.([2.5]) determines the chemical potential. In the latter case, the fluctuation contribution to $\mathscr{S}$ has to be restored to determine the critical temperature at a given density. 
The hyperbolic tangent function in (2.5) may be approximated by one with $T<<|\chi|$ and we replace the coupling constant by the $s$-wave scattering length via $1 / g-1 / V \sum_{\mathbf{k}}\left(2 \varepsilon_{\mathbf{k}}\right)^{-1} \equiv$ $-m / 4 \pi a_{s}$. Carrying out the summation explicitly, we find that

$$
-\frac{m}{4 \pi a_{s}}=\frac{\sqrt{\omega_{B}} m^{3 / 2}}{4 \sqrt{2} \pi} \zeta\left(\frac{1}{2}, \frac{|\chi|}{\omega_{B}}\right)
$$

with the Hurwitz zeta function defined by $\zeta(s, a)=\sum_{n=0}^{\infty}(n+a)^{-s}$. In the absence of magnetic field, the RHS of ([2, becomes $-m^{3 / 2} \sqrt{|\chi|} /(2 \sqrt{2} \pi)$ and we have a solution $\chi=-1 /\left(2 m a_{s}^{2}\right)$ only for $a_{s}>0$, which defines the strong coupling domain. The weak coupling domain, $a_{s}<0$, however, entirely resides on the BCS side of the BCS/BEC crossover. When the magnetic field is turned on, the RHS of (2.6) can take both signs and a solution emerges in the weak coupling domain. This is caused by the dimensional reduction of the Landau orbitals, i.e., magnetic catalysis and the BEC limit can be approached in both strong and weak coupling domains.

The eq.(2.6) sets the chemical potential at the energy of a bound pair of zero center-of-mass momentum in vacuum and this is the condition for the BEC of an ideal Bose gas. The contributions of the bound pairs of nonzero momentum, however, is ignored here. Therefore the mean field approximation is not sufficient and the contribution from the bound pairs with nonzero momenta to the density equation has to be restored to determine the transition temperature.

The Gaussian fluctuations about the trivial saddle point $\Delta_{0}=0$ can be taken into account by replacing the entire $\mathscr{S}$ of $\left(\mathbb{2}_{3}\right]$ ) by its expansion to the quadratic order in the entire boson field $\Delta(x)$.

$$
\mathscr{S}_{\text {eff }}[\Delta] \simeq \mathscr{S}[0]-\int d \tau d^{3} \mathbf{x} \frac{|\Delta(x)|^{2}}{g}-\int d \tau d \tau^{\prime} d^{3} \mathbf{x} d^{3} \mathbf{x}^{\prime}\left[G_{+}\left(x, x^{\prime}\right) \Delta\left(x^{\prime}\right) G_{-}\left(x^{\prime}, x\right) \Delta^{*}(x)\right],
$$

with $G_{ \pm}^{-1}\left(x, x^{\prime}\right)$ the diagonal elements in (2.4).

In terms of the Fourier transformation,

$$
\mathscr{S}_{\text {eff }}[\Delta]=\mathscr{S}_{\text {eff }}[0]+\sum_{\omega_{n_{p}}, \mathbf{p}} \Gamma^{-1}\left(i \omega_{n_{p}}, \mathbf{p}\right)\left|\Delta\left(i \omega_{n_{p}}, \mathbf{p}\right)\right|^{2}
$$

where the dependence of the coefficient $\Gamma^{-1}\left(i \omega_{n_{p}}, \mathbf{p}\right)$ on $T, \mu$ and $\mathbf{B}$ has been suppressed and the thermodynamic potential density reads

$$
\Omega=\Omega_{0}-\frac{1}{\beta V} \sum_{\omega_{n_{p}}, \mathbf{p}} \ln \Gamma\left(i \omega_{n_{p}}, \mathbf{p}\right) .
$$

where $\Omega_{0}=-2 /(\beta V) \sum_{k_{y}, k_{z} ; l} \ln \left[1+\exp \left(\varepsilon_{k_{z}}+l \omega_{B}-\chi\right)\right]$ is the thermodynamic potential of an ideal Fermi gas. It follows that

$$
n=n_{0}-\frac{1}{\beta V} \frac{\partial}{\partial \mu} \sum_{\omega_{n_{p}}, \mathbf{p}} \ln \Gamma\left(i \omega_{n_{p}}, \mathbf{p}\right),
$$

with $n_{0}=2 V \sum_{k_{y}, k_{z} ; l}\left[\exp \left(\beta\left(\varepsilon_{k_{z}}+l \omega_{B}-\chi\right)+1\right]^{-1}\right.$ the fermionic contribution to the density. Continuating $i \omega_{n_{p}}$ to an arbitrary real frequency $\omega$ according to the prescription in [25] and introducing a phase shift defined by $\Gamma(\omega \pm i 0, \mathbf{q})=|\Gamma(\omega, \mathbf{q})| \exp [ \pm i \delta(\omega, \mathbf{q})]$, the number equation can also be written as [20]

$$
n=n_{0}+\frac{1}{V} \sum_{\mathbf{q}} \int_{-\infty}^{\infty} \frac{d \omega}{\pi} n_{B}(\omega) \frac{\partial \delta}{\partial \mu}(\omega, \mathbf{q}) .
$$


with $n_{B}(\omega)=\left(e^{\beta \omega}-1\right)^{-1}$ the Bose-Einstein distribution function. The pair of equations, ([2.6) and (‥CI), at zero magnetic field are widely employed in the context of BCS/BEC crossover in the literature.

Through some calculations, we obtain

$$
\Gamma^{-1}(\omega, \mathbf{q}) \simeq a_{1}\left[\omega+2(\mu-\bar{\mu})-\frac{p_{z}^{2}}{4 m}\right]-a_{2} \frac{p_{\perp}^{2}}{4 m} .
$$

with

$$
a_{1}=\frac{m^{3 / 2}}{16 \pi \sqrt{2 \omega_{B}}} \sum_{l=0}^{\infty}\left(l+\frac{|\bar{\chi}|}{\omega_{B}}\right)^{-\frac{3}{2}}
$$

and

$$
a_{2}=\frac{m^{3 / 2}}{\pi \sqrt{2 \omega_{B}}} \sum_{l=0}^{\infty}\left(\frac{l+\frac{1}{2}}{\sqrt{l+\frac{|\bar{\chi}|}{\omega_{B}}}}-\frac{l}{2 \sqrt{l-\frac{1}{2}+\frac{|\bar{\chi}|}{\omega_{B}}}}-\frac{l+1}{2 \sqrt{l+\frac{1}{2}+\frac{|\bar{\chi}|}{\omega_{B}}}}\right) .
$$

where the frequency $\omega$ is the continuation of the Matsubara frequency $i \omega_{n_{p}}$ to the neighborhood of the zero and $\bar{\chi}$ is the solution to the mean field equation (2.6). Obviously, the kinetic term becomes anisotropic with respect to the directions along and perpendicular to the magnetic field due to the rotational symmetry breaking by the magnetic field.

The partition function (2.2) under the Gaussian approximation of fluctuations may be written as

$$
\mathscr{Z}=\mathscr{N} \int \mathscr{D} \phi^{*} \mathscr{D} \phi \exp \left\{\sum_{\omega_{n_{p}}, \mathbf{p}} \phi_{p}^{*}\left(\omega-\omega_{b}+2 \mu\right) \phi_{p}\right\} .
$$

where $\phi$ is the rescaled field of the fluctuation $\Delta$ and $\omega_{b}=-E_{B}+\omega_{B}+p_{z}{ }^{2} /(4 m)+\kappa p_{\perp}^{2} /(4 m)$ is the dispersion relation with $E_{B}=-2 \bar{\chi}$ the binding energy that is measured from the lowest Landau level. We have also the explicit expression of the anisotropy factor

$$
\kappa \equiv a_{2} / a_{1}=16 \frac{\zeta\left(-\frac{1}{2}, \frac{|\bar{\chi}|}{\omega_{B}}\right)-\zeta\left(-\frac{1}{2}, \frac{1}{2}+\frac{|\bar{\chi}|}{\omega_{B}}\right)+\left(\frac{1}{2}-\frac{|\bar{\chi}|}{\omega_{B}}\right)\left[\zeta\left(\frac{1}{2}, \frac{|\bar{\chi}|}{\omega_{B}}\right)-\zeta\left(\frac{1}{2}, \frac{1}{2}+\frac{|\bar{\chi}|}{\omega_{B}}\right)\right]}{\zeta\left(\frac{3}{2}, \frac{|\bar{\chi}|}{\omega_{B}}\right)} .
$$

One can show that $\kappa \leq 1$ for an arbitrary value of the ratio $|\bar{\chi}| / \omega_{B}$ and is a monotonically increasing function of this ratio.

The partition function (2.15) is nothing but an ideal Bose gas with anisotropy in kinetic term. The density is dominated by the pole of $\frac{\partial \delta}{\partial \mu}$ of (‥JI) at $\omega=\omega_{b}-2 \mu$. The condensation temperature is determined by setting the chemical potential at the solution of the mean field equation ([2. $)$, i.e. $\mu=\omega_{B} / 2+\bar{\chi}$, and we have

$$
n=2 \int \frac{d^{3} \mathbf{p}}{(2 \pi)^{3}}\left[\exp \left(\frac{p_{z}^{2}+\kappa p_{\perp}^{2}}{4 m T_{c}}\right)-1\right]^{-1}
$$

It follows that

$$
T_{c}=\kappa^{\frac{2}{3}} T_{c}^{0}
$$

where

$$
T_{c}^{0}=\left[\frac{n}{2 \zeta(3 / 2)}\right]^{2 / 3} \frac{\pi}{m} .
$$

is the condensation temperature of an ideal Bose gas of the same density at zero magnetic field. 


\section{Bose-Einstein condensation in a magnetic field}

In this section, we shall explore the magnetic field dependence of the BEC temperature (2.18) for both strong coupling, $a_{s}>0$ and weak coupling, $a_{s}<0$.

We shall begin with an examination of the two asymptotic behaviors $r \gg 1$ and $r \ll 1$ of the Hurwitz zeta function $\zeta(s, r)$. The large $r$ expansion reads

$$
\zeta(s, r) \simeq \frac{r^{-s+1}}{s-1}+\frac{r^{-s}}{2}+\frac{s r^{-s-1}}{12}-\frac{s(s+1)(s+2) r^{-s-3}}{720}+O\left(r^{-s-5}\right) .
$$

The negative value of $\zeta(1 / 2, r)$ in this limit leads us to the strong coupling domain via the meanfield equation (2.6)

$$
\frac{1}{a_{s}} \simeq \sqrt{2 m|\bar{\chi}|}-\frac{1}{2} \sqrt{\frac{m}{2|\bar{\chi}|}} \omega_{B}>0
$$

If follows that the approximate binding energy $|\bar{\chi}| \simeq\left(1+e B a_{s}^{2}\right) /\left(2 m a_{s}^{2}\right)$, with $a_{s}<<1 / \sqrt{e B}$. The anisotropy factor (2.16) reads

$$
\kappa \simeq 1-\frac{1}{16}\left(\frac{\omega_{B}}{|\bar{\chi}|}\right)^{2} \simeq 1-\frac{1}{4}(e B)^{2} a_{s}^{4} .
$$

and gives rise to a slight suppression of the condensation temperature according to (2.]8), corresponding to an inverse magnetic catalysis.

The small $r$ behavior follows from the relation

$$
\zeta(s, r)=r^{-s}+\zeta(s, 1+r) \simeq r^{-s}+\zeta(s)
$$

which, for $s>0$, is dominated by the first term on RHS and corresponds to the lowest Landau level approximation in our problem. The positivity of $\zeta\left(\frac{1}{2}, \frac{|\bar{\chi}|}{\omega_{B}}\right)$ in this case, i.e. $|\bar{\chi}|<<\omega_{B}$, together with the mean-field equation (12.6) implies a negative $a_{s}$ and thereby the weak coupling domain, i.e.

$$
\frac{1}{a_{s}} \simeq-\sqrt{\frac{m}{2|\bar{\chi}|}} \omega_{B}<0
$$

It follows that the binding energy $|\bar{\chi}| \simeq \frac{1}{2} m \omega_{B}^{2} a_{s}^{2}$ is entirely induced by the magnetic field, as a consequence of the magnetic catalysis. In terms of the binding enery, the inequality $|\bar{\chi}|<<\omega_{B}$ implies $\left|a_{s}\right|<<\frac{1}{\sqrt{e B}}$. The anisotropy factor

$$
\kappa \simeq 8 \frac{|\bar{\chi}|}{\omega_{B}} \simeq 4 e B a_{s}^{2}<<1 .
$$

in this case and maximizes the suppression of the condensation temperature.

Since $\zeta(1 / 2, r)$ is a monotonically decreasing function of $r$ and is negative (positive) for a large (small) $r$, its zero, $r_{c}$, serves a demarcation between the strong coupling domain, where $a_{s}>0$ and $|\bar{\chi}| / \omega_{B}>r_{c}$, and the weak coupling domain, where $a_{s}<0$ and $|\bar{\chi}| / \omega_{B}<r_{c}$. The value of $r_{c}$ as well as the solution of the mean-field equation (2.6) and the condensation temperature for $|\bar{\chi}| / \omega_{B}=O(1)$ can only be calculated numerically. We find $r_{c} \simeq 0.303$ and $|\bar{\chi}| \simeq r_{c} \omega_{B} \simeq 0.303 \omega_{B}$ as $B \rightarrow \infty$. 


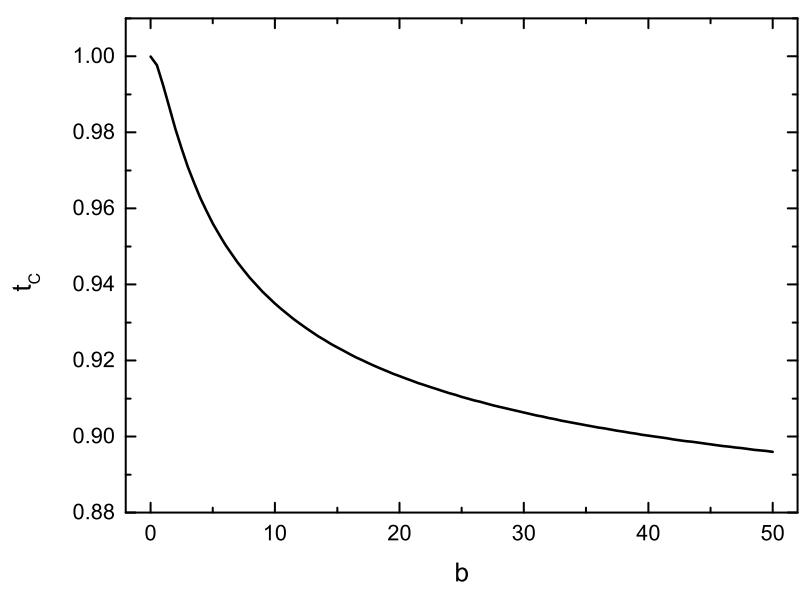

Figure 1: The ratio of $\mathrm{BE}$ condensation temperature $t_{c}$ versus the dimensionless magnetic field $b$ in strong coupling domain.

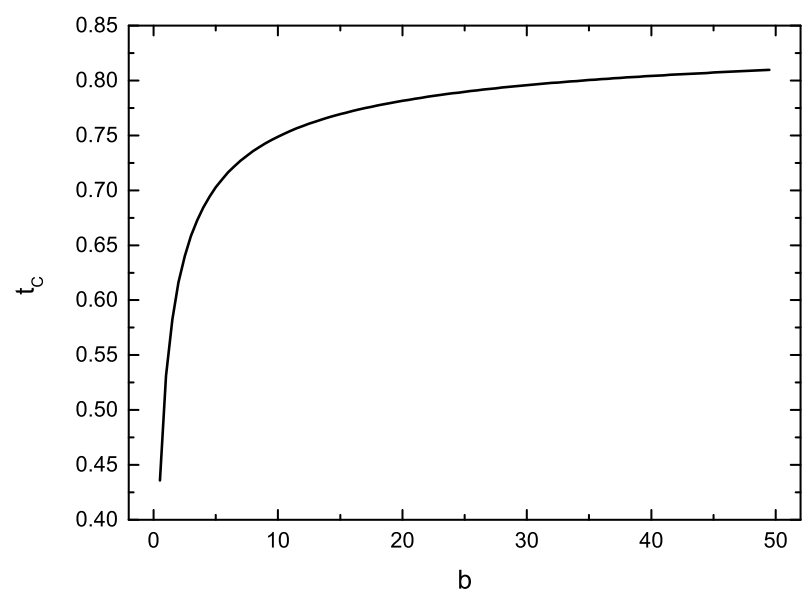

Figure 2: The ratio of BE condensation temperature $t_{c}$ versus the dimensionless magnetic field $b$ in weak coupling domain.

In the strong coupling domain, $a_{s}>0$, bound pairs exist in the absence of magnetic field with the binding energy $E_{b}=1 /\left(m a_{s}^{2}\right)$ and condense at the temperature $T_{c}^{0}$. The mean-field equation (2.6) and the condensation temperature (2.18) in a magnetic field can be expressed in terms of dimensionless quantities, i.e.

$$
b^{-\frac{1}{2}}=-\frac{1}{2} \zeta\left(\frac{1}{2}, \frac{v}{b}\right)
$$

and

$$
t_{c}=\kappa^{\frac{2}{3}}\left(\frac{v}{b}\right)
$$


where $b \equiv \frac{\omega_{B}}{E_{b}}, v \equiv \frac{|\bar{\chi}|}{E_{b}}$ and $t_{c} \equiv \frac{T_{c}}{T_{c}^{0}}$. The solution of (3.7) for $t_{c}$ versus the dimensionless magnetic field $b$ is plotted in Fig.1. The condensation temperature deceases as magnetic field increases, consistent with the large $r$ limit. The physical reason for this inverse magnetic catalysis is the enhanced fluctuations by the anisotropic distortion of the bosonic spectrum, $\kappa<1$, in the magnetic field. The effect is, however, rather mild with $\kappa$ decreasing from one at $b=0$ to about 0.9 at $b=50$ because the ratio $r$ never drops to a level to warrant the LLL approximation within the strong coupling domain.

In the weak coupling domain, $a_{s}<0$, bound pairs are formed through the mechanism of magnetic catalysis. The mean-field equation becomes

$$
b^{-\frac{1}{2}}=\frac{1}{2} \zeta\left(\frac{1}{2}, \frac{v}{b}\right)
$$

with the sign on RHS opposite to that of (B.]). The formula for the condensation temperature, (B.8), remains unchanged. Here the denominator of $b$ and $t_{c},\left|E_{b}\right|$ and $T_{c}^{0}$, do not carry direct physical meaning other than reference scales because the bound pairs do not exist in the absence of magnetic field. The condensation temperature in Fig. 2 increases as magentic field increases, which is consistent with the analysis in small $r$ limit. The LLL approximation works in the limit $r \rightarrow 0$, where the anisotropy of the bosonic spectrum is maximized. An increasing magnetic field raises the ratio $r$ and promotes the contribution from higher LL's, and thereby increases the condensation temperature.

Notice that, however, the condensation temperature is always suppressed compared with that of an ideal Bose gas of mass $2 m$ regardless of the coupling strength because of the inequality $\kappa<1$ for all real $a_{s}$.

Before concluding this section, we would like to comment on the validity of the Gauss approximation of fluctuations in the context of the BEC limit, which ignored the quartic and higher powers on $\Delta(x)$ in $([2.3)$. These terms represents the interactions among the Cooper pairs, which becomes significant when their wave functions overlap. Therefore the approximation will deteriorate at the density at which the inter-particle distance $n^{-1 / 3}$ becomes comparable to the size of the bound pairs.

\section{Conclusion}

We have investigated a system of nonrelativistic bound pairs made of oppositely charged fermions in the presence of an external magnetic field. We found that the variation of the BEC temperature with respect to the magnetic field depends on the coupling strength of pairing. In strong coupling domain where the bound pairs(composite bosons) exist already without magnetic field, we found the inverse magnetic catalysis that the condensation temperature decreases as increasing magnetic field. In weak coupling domain where the bound pairs are induced by magnetic field, the transition temperature exhibits the usual magnetic catalysis effect. In either domain, the condensation temperature is lower than that of an ideal Bose gas of the same mass, $2 m$, of each particle in the absence of magnetic field. The suppression effect is maximized when the lowest Landau Level approximation works which requires the ratio of binding energy relative to the lowest Landau level over the spacing between adjacent Landau levels, $r=|\chi| / \omega_{B}<<1$. This condition is realized in 
the weak coupling domain under a weak magnetic field. Otherwise, the ratio is order $O(1)$ and the suppression effect is less pronounced. In particular, the binding energy diverges like $|\chi| \simeq 0.303 \omega_{B}$ in the strong field limit, for both strong and weak couplings, making the ratio 0.303 with the suppression factor $\kappa \simeq 0.792$. Of course, the BEC approximation requires the fermion density of the system to be sufficiently low such that the bound pairs do not overlap. With increasing density, individual bound pairs lose their identities and BCS condensation emerges. This crossover within the weak coupling domain under a magnetic field is what we called the magnetic field induced BCS/BEC crossover. Without the magnetic field, the weak coupling domain corresponds only to the BCS side of the crossover.

To simplify the calculation, we ignored the spin degrees of freedom of the fermions as they do not contribute to the pairing dynamics. The contribution of bound pairs with different spin configurations to the total density are weighted by the Bose-Einstein distribution function with different Zeeman energies. For the temperature much lower than the Zeeman energy of a fermion, the density is dominated by the pairing channel with the lowest Zeeman energy and our previous results can be carried over.

The system considered in the present work is of theoretical value only at this stage, but the physics involved may be relevant to the BEC of excitons in a semiconductor in an magnetic field [26]or the magnetic color-flavor-locked superfluidity of a dense quark matter [27]. In the former case, the pairing is implemented via the long range Coulomb attraction (the scattering length formulation needs to be modified accordingly) and we are always in the strong coupling domain. In the later case, the pairing force stems from the non-perturbative QCD effect and the Coulomb interaction becomes perturbative at the typical density of a quark matter.

\section{Acknowledgments}

B. F. is supported by NSFC under grant No. 11305067 and the Fundamental Research Funds for the Central Universities, HUST: No. 2013QN015. D. Hou and H.C. Ren are partly supported by NSFC under Grant Nos. 11375070, 11135011 and 11221504.

\section{References}

[1] D. E. Kharzeev, K. Landsteiner, A. Schmitt and H-u Yee, Lect. Notes Phys. 871, 1 (2013) and references therein.

[2] K. Klimenko, Theor. Math. Phys. 89, 1161 (1991); Z. Phys. C 54, 323 (1992).

[3] V. Gusynin, V. Miransky and I. Shovkovy, Phys. Rev. Lett. 73, 3499 (1994); Phys. Rev. D 52, 4718 (1995); Phys. Lett. B 349, 477 (1995); Nucl. Phys. B 462, 249 (1996).

[4] K. Fukushima, M. Ruggieri and R. Gatto, Phys. Rev. D 81, 114031 (2010).

[5] A. J. Mizher, M. Chernodub and E. S. Fraga, Phys. Rev. D 82, 105016 (2010).

[6] V. Skoko, Phys. Rev. D 85, 034026 (2012).

[7] G. Bali, F. Bruchmann, G. Endrődi, Z. Fodor, S.D. Katz, S. Krieg, A. Schäfer and K.K. Szabó, J. High Energy Phys. 02, (2012) 044. 
[8] G. Bali, F. Bruchmann, G. Endrődi, Z. Fodor, S.D. Katz, S. Krieg and A. Schäfer, Phys. Rev. D 86, 071502 (2012).

[9] F.Preis, A. Rebhan and A. Schmitt, J. High Energy Phys. 03 (2011) 033.

[10] K. Fukushima and J. M. Pawlowski, Phys. Rev. D 86, 076013 (2012).

[11] K. Fukushima and Y. Hidaka, Phys. Rev. Lett. 110, 031601 (2013).

[12] T. Kojo and N. Su, Phys. Lett. B 720, (2013)

[13] F. Bruckmann, G. Endrodi, and T. G. Kovacs, J. Hign Energy Phys. 1304, 112 (2013).

[14] J. Chao, P. Chu and M. Huang, Phys. Rev D 88, 054009 (2013); L. Yu, H. Liu and M. Huang, Phys. Rev. D 90, 074009 (2014);L. Yu, J. Doorsselaere and M. Huang, arXiv:1411.7552.

[15] E.J.Ferrer, V.de la Incera and X. Wen, arXiv:1407.3503.

[16] R.Farias, K.Gomes, G. Krein and M. Pinto, arXiv:1404.3931.

[17] N. Mermin and H. Wagner, Phys. Rev. Lett. 17, 1133 (1966);P. Hohenberg, Phys. Rev. 158, 383 (1967); S.Coleman, Commun. Math. Phys. 31, 259 (1973).

[18] B. Feng, D-f Hou and H-c Ren, In preparation.

[19] P. Noziéres and S Schmitt-Rink, J. Low Temp. Phys. 59, 195 (1985).

[20] C. Sá de Melo, M. Randeria and J.R.Engelbrecht, Phys. Rev. Lett. 71, 19 (1985).

[21] Y. Nishida and H. Abuki, Phys. Rev. D 72, 096004 (2005); H. Abuki, Nucl. Phys. A 791, 117 (2007);H. Abuki, G. Baym, T. Hatsuda and Y. Yamamoto, Phys. Rev. D 81, 125010 (2010).

[22] J. Deng, A. Schmitt and Q. Wang, Phys. Rev. D 76, 034013 (2007).

[23] J. Wang, V. de la Incera, E.J. Ferrer and Q. Wang, Phys. Rev. D 84, 065014 (2011); E. J. Ferrer and J. Keith, Phys. Rev. C 86 035205; E.J. Ferrer, V. de la Incera, I. Portillo and M. Quiroz, Phys. Rev. D 89 (2014) 085034; E.J. Ferrer, V. de la Incera, J. Keith and I. Portillo, arXiv:1405.7422.

[24] G. Sun, L. He and P. Zhuang, Phys. Rev. D 75, 096004 (2007);L. He and P. Zhuang, Phys. Rev. D 76, 056003 (2007).

[25] G. Baym and N. D. Mermin, J. Math. Phys., 2, 232 (1961).

[26] J.Eisenstein, Science 305, 950 (2004); J.Eisenstein and A.H. MacDonald, Nature 432, 691 (2004).

[27] E.J. Ferrer, V. de la Incera, Phys. Rev. Lett. 97, 22301 (2007); E.J. Ferrer, V. de la Incera, Phys. Rev. D 76, 045011 (2007);B. Feng, E. J. Ferrer and V. de la Incera, Nucl. Phys. B 853213 (2011). 